\title{
Architecture for Raw Data Processing at Sensor Nodes in Semantic Wireless Sensor Networks
}

\author{
Udai Arora \\ Manipal Institute of Technology \\ Manipal \\ Karnataka
}

\author{
Archana Kumar \\ Manipal Institute of Technology \\ Manipal \\ Karnataka
}

\begin{abstract}
More and more WSNs of diverse capabilities are being deployed so as to get data about the physical or environmental conditions they monitor. Even though a large amount of data is produced by these sensor networks, it is generally heterogeneous and un-semantic in nature thereby hindering collaboration between different WSNs. In this paper, we capitalize on the concept of semantic web and explore the use of associating these semantics with this raw data so as to facilitate easy integration of different WSNs, thereby resulting in better experience for the user. We process this data at the sensing node level rather than at gateway node level and provide a theoretical architecture of the sensor node to facilitate this.
\end{abstract}

\section{General Terms}

Semantic Networks, Wireless Sensor Networks, Data processing at sensor node

\section{Keywords}

Wireless Sensor Network, Semantic Web, Semantic Sensor Networks, Data Processing

\section{INTRODUCTION}

Wireless sensor network has been an area of top interest among the researchers over the past decade. This is mainly due to the large number of usages it offers- from real world monitoring to location based services and its innumerable potential application- from gaming to future home systems. WSN monitor conditions at different locations, such as temperature, humidity, vehicular movement, lightning condition, pressure, soil makeup, noise levels, the presence or absence of certain kinds of objects, mechanical stress levels on attached objects, the current characteristics such as speed, direction and size of an object [1].

While most of the research on WSN has focused on deployment or localization, the data generated by these sensor networks has got a little attention. We are generally not interested in raw data generated by wireless sensor networks. Rather, we want interested in higher level events. For example, consider a greenhouse consisting of 2 wireless sensor networks- one to measure the temperature and another to measure the humidity. Suppose we want to open the vents when the temperature and humidity drops below a certain level. In this case we are only interested in higher level event rather than the raw data generated by the nodes of the two sensor networks.
Currently, the processing of data is mostly done in the more powerful nodes called gateway nodes- the nodes responsible for relaying data to the application. The sensor nodes are used only for capturing data. This leads to a lot of energy being wasted at sensor nodes.

This paper is organized as follows: Section II introduces the use of semantic web technologies in wireless sensor networks. Section III presents an architecture for the sensing node followed by the conclusion and future work in the final section- Section IV

\section{WIRELESS SENSOR NETWORKS AND SEMANTIC WEB}

A wireless sensor network is a group of specialized transducers with a communication infrastructure intended to monitor physical phenomena like temperature, sound, light intensity, location, motion of objects and so on[2]. They communicate with each other over the air with the help of an embedded radio. Generally, the node size in a particular WSN ranges from a few hundred to a couple of thousand nodes. WSNs are highly application specific due the constraints such as limited range, low processing capability, etc. Each sensor network is deployed to serve a specific purpose and uses its own protocols. This heterogeneity in sensor networks makes it impossible to communicate with each other or to reuse and share their data with different applications. Hence, it is necessary for the data produced by wireless sensor nodes to have semantics so as to enable cooperation.

An ontology based description is provided by Semantic Web Services aiming to automate the discovery, composition, invocation and monitoring of web services and thereby allowing the relationship between different sets of data and their properties to be machine-processable. The semantic web allows the possibility of associating a parameter to a concept rather than just to a datatype- which is the case with the syntactic web.

Six years ago, Tim Berners-Lee, James Hendler and Ora Lassila unveiled a nascent vision of the Semantic Web: a highly interconnected network of data that could be easily accessed and understood by any desktop or handheld machine. They painted a future of intelligent software agents that would head out on the World Wide Web and automatically book flights and hotels for our trips, update our medical records and give us a single, customized answer to a particular question without our having to search for information or pore through results[4].

Motivated by the concept of semantic web, semantics are now being incorporated in the data produced by these nodes and thereby enabling "machine-to-machine" communication. 
The following diagram shows an architecture for the same:

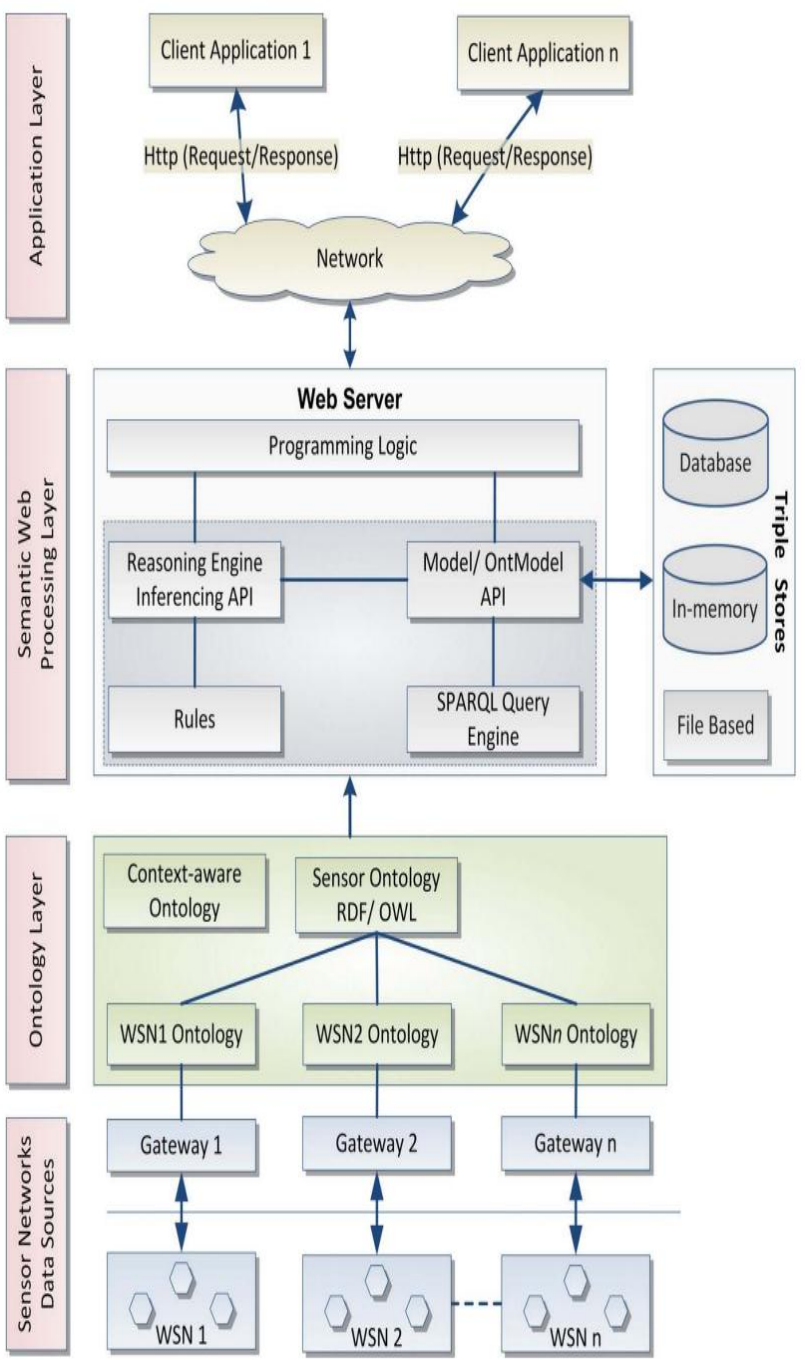

Fig 1: A semantic web architecture for WSN

The above architecture proposed in [2] is a cross layered architecture where the semantic routing protocols are more robust and the semantics simplify data processing and interpretation thereby making the solution of critical problems feasible.

\section{ENABLING DATA PROCESSING AT SENSOR NODE- AN ARCHITECTURE}

\subsection{Current Problem}

The in-network data processing can be done in homogenous networks only. Ontologies are presently used to tackle this problem and are used in heterogeneous networks. However, the processing is limited to gateway nodes and sensor nodes do not generally participate in the processing. As a result, the data needs to be sent from the sensor nodes to the gateway nodes for processing to be done. We deal with this problem by enabling the sensor nodes to process the raw produced by the various sensing nodes and take an action accordingly.

\subsection{Sensor node architecture}

As discussed above, the analysis and processing of raw data from the various heterogeneous networks takes place at the gateway node level. The following diagram presents an abstract architecture in which the processing can be performed at the sensor node itself:

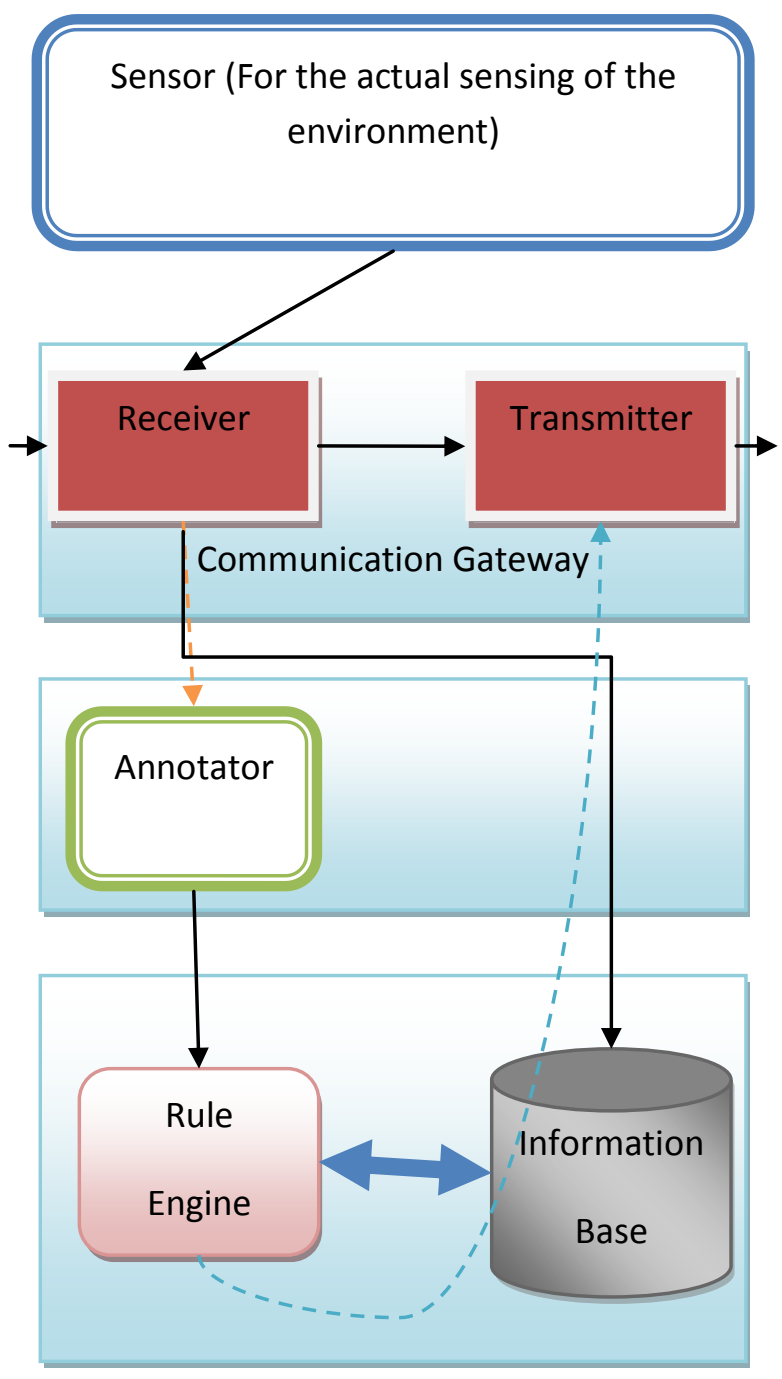

Fig 2: Sensor node architecture for semantic sensor node where processing takes place at sensor node itself

The sensor senses the physical environment and sends the data to the receiver. The receiver forwards the data to the annotator where the data is annotated with semantics and is sent into the rule engine. The rule engine can have communication with the information base. The information base contains the data recorded by sensors. The sensed message is then shared and might even generate a trigger if there is a match is found in the rule engine.

- Share: A share message received by the receiver is used to update the information base of a node and is forwarded for further sharing by the transmitter.

- Trigger: A trigger message is a high priority message generated by a node when there is a complete match. This message is forwarded to the base station informing it that an event has occurred.

With the above node architecture, the nodes are able to communicate with each other, even if they don't belong to the same sensor network. This also enables these nodes to process the raw data produced by the various sensor nodes and take an action depending on a match with a rule in rule engine. 


\section{CONCLUSION AND FUTURE WORK}

Multiple sensor networks may be deployed to monitor an area The data produced by these networks is heterogeneous in nature and hence hinders communication between different networks. Semantics can be associated with sensor data to enable communication between heterogeneous WSN. The processing and analysis of this data takes place at gateway nodes. With the architecture presented, this processing can be performed at the sensor node level itself, thereby improving performance and saving energy.

.For future research, we will quantitatively evaluate the performance with this new architecture and draw out comparisons with the traditional one. We will also try to make the sensor nodes more context-aware. We will also evaluate the effect of this architecture on the lifetime of the sensor network.

\section{REFERENCES}

[1] Ying Miao 2005. APPLICATIONS OF SENSOR NETWORKS.

[2] Vincent Huang, Muhammad Kashif J. 2008 Semantic Sensor Information Description and Processing. In Proceedings of The Second International Conference on Sensor Technologies and Applications.

[3] Gian Ricardo Berkenbrock, Celso Massaki Hirata, Frederico Guilherme Alvares de Oliveira Junior, Jos' Maria Parente de Oliveira. 2009. Applying Semantic Web Services and Wireless

[4] Lee Feigenbaum, P. 2007 The Semantic Web in action

[5] Minglu Li 2005 A Semantic based architecture for sensor networks.

[6] Liyang Yu 2011. A developer's guide to the semantic web.
[7] Benjamin Szekely, Elias T. 2004. A Semantic Data Collection Model for Sensor Network Applications.

[8] Sheth, A. P. 1999 "Changing Focus on Interoperability in Information Systems: From System, Syntax, Structure to Semantics". In M. Goodchild, M. Egenhofer, R. Fegeas, and C. Kottman, Interoperating Geographic Information System, pp. 5-30. Kluwer Academic Publishers.

[9] Vincent Huang and Mattias Johansson, 2007 "Usage of semantic web technologies in a future M2M communication system", Proceedings of the 1st European Semantic Technology Conference, Vienna, Austria

[10] Moodley, D., and Simonis, I. 2006 "A New Architecture for the Sensor Web: The SWAP Framework". A workshop of the 5th International Semantic Web Conference ISWC

[11] ] Jung-sick Byun, Woo-Suk Shim, Won-Kee Hong, "WSNbased intelligent telematics system", ECBS 2006 13th Annual IEEE International Symposium and Workshop on Engineering of Computer Based Systems, Pages: 2 pp.

[12] Wache, H., Vogele, T., Visser, U., Stuckenschmidt, H., Schuster, G., Neumann, H., et al. "Ontology-based integration of information - a survey of existing approaches". Proceedings of the IJCAI-01 Workshop: Ontologies and Information Sharing, 2001.

[13] G. Antoniou and F. van Harmelen, 2004. A Semantic Web Primer (Cooperative Information Systems). The MIT Press

[14] W3C Semantic Web Activity, http://www.w3.org/2001/sw/ 